Cinémas

Revue d'études cinématographiques

Journal of Film Studies

\title{
Le Documentaire autoréflexif au Québec. L'Émotion dissonante et Passiflora
}

\section{Ian Lockerbie}

Volume 4, numéro 2, hiver 1994

Le Documentaire

URI : https://id.erudit.org/iderudit/1001026ar

DOI : https://doi.org/10.7202/1001026ar

Aller au sommaire du numéro

Éditeur(s)

Cinémas

ISSN

1181-6945 (imprimé)

1705-6500 (numérique)

Découvrir la revue

Citer cet article

Lockerbie, I. (1994). Le Documentaire autoréflexif au Québec. L'Émotion dissonante et Passiflora. Cinémas, 4(2), 118-132.

https://doi.org/10.7202/1001026ar
Résumé de l'article

L'article traite de l'équilibre précaire dans le documentaire entre le projet social et les préoccupations formelles. Tandis que les pressions institutionnelles (spécialement en télévision) ont de plus en plus conduit le genre en direction de l'actualité/la transparence, certains cinéastes du Québec ont, au contraire, cultivé les dimensions formelles/esthétiques au point de frôler l'hermétisme. Une telle autoréflexion formelle apparaît comme un contrepoids nécessaire aux pressions normalisantes et a produit des résultats significatifs dans des films comme Le Dernier Glacier, L'Émotion dissonante et Passiflora. 


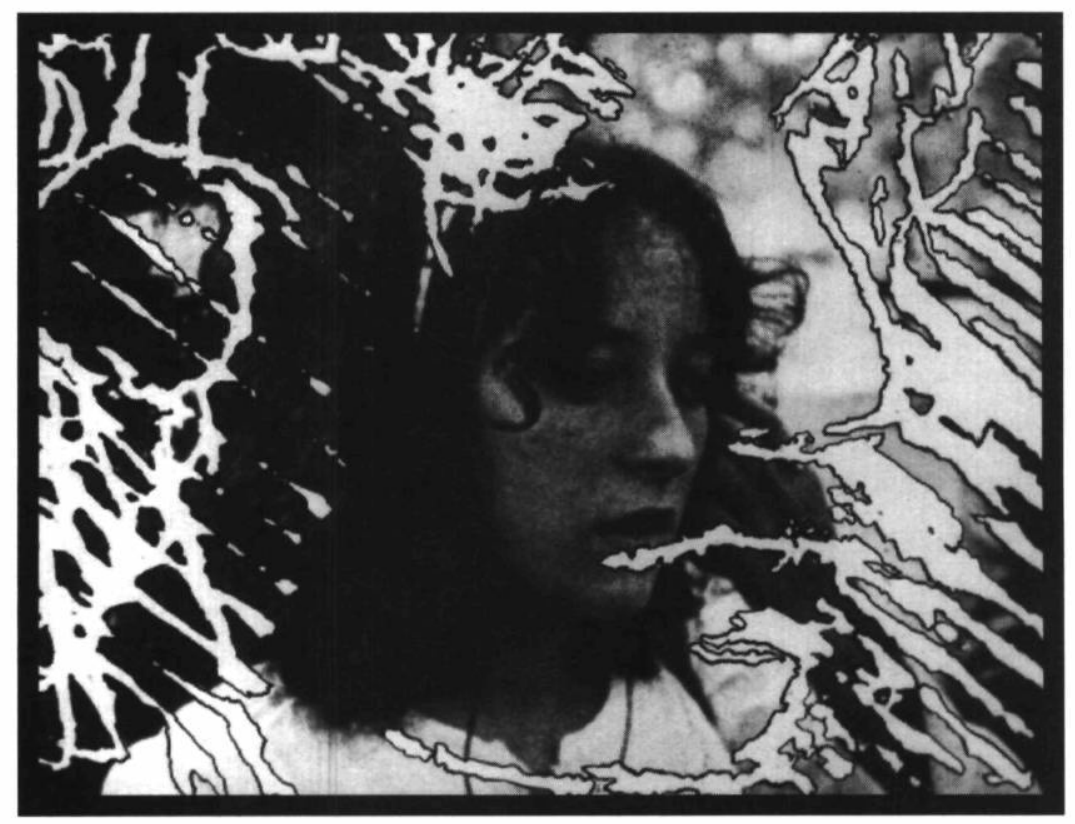

L'EMOTION DISSONANTE de Fernand Bélanger (1984) Coll. Cinémathèque québécoise 


\title{
Le Documentaire autoréflexif au Québec. L'Émotion dissonante et Passiflora ${ }^{1}$
}

\section{Ian Lockerbie}

\author{
RÉSUMÉ
}

L'article traite de l'équilibre précaire dans le documentaire entre le projet social et les préoccupations formelles. Tandis que les pressions institutionnelles (spécialement en télévision) ont de plus en plus conduit le genre en direction de l'actualitélla transparence, certains cinéastes du Québec ont, au contraire, cultivé les dimensions formelles/esthétiques au point de frôler l'hermétisme. Une telle autoréflexion formelle apparaît comme un contrepoids nécessaire aux pressions normalisantes et a produit des résultats significatifs dans des films comme Le Dernier Glacier, L'Émotion dissonante et Passiflora.

\section{ABSTRACT}

The article discusses the precarious balance in documentary between social project and formal concerns. While institutional pressures (especially in television) have increasingly driven the genre towards actuality/transparency, some Quebec film makers have, on the contrary, cultivated the formal/aesthetic dimensions to the point of hermeticism. It is argued that such formal self-consciousness is a necessary counterweight to normalising pressures and has yielded significant results in films such as Le Dernier Glacier, L'Émotion dissonante and Passiflora. 
Le documentaire joue dans la vie et la culture du Canada un rôle plus important que partout ailleurs, grâce surtout à la présence de l'Office national du film, fondé en 1939 par John Grierson. Bien que l'ONF ait acquis une réputation mondiale dans d'autres domaines - notamment celui du film d'animation, où les œuvres de Norman McLaren constituent son plus grand titre de gloire - , sa raison d'être a toujours été la production de documentaires. De plus, sa prééminence dans ce rôle a permis à un nombre considérable de producteurs indépendants du secteur privé de se manifester, au Québec non moins que dans les autres provinces.

Pour Grierson, le documentaire devait contribuer à instruire le public, d'abord en l'informant sur les grands problèmes sociaux de l'heure, et ensuite en inspirant aux citoyens, par une meilleure compréhension de leurs semblables, le désir de participer davantage à la vie de leur collectivité. C'était bien ainsi du reste que la Loi sur le cinéma — d'où est né l'ONF - concevait la mission de l'Office : dire aux Canadiens qui ils sont, et dire au monde ce qu'est le Canada.

En même temps, Grierson affirmait que, pour atteindre ses objectifs, le documentaire devait être une forme d'art. Son intérêt pour le documentaire tenait essentiellement à sa conviction que, dans des sociétés aussi complexes que celles du $\mathrm{XX}^{\mathrm{e}}$ siècle, le citoyen moyen ne saisirait les problèmes importants que s'ils lui étaient présentés d'une façon vivante et imaginative, avec toute l'intensité que pouvait leur conférer la puissance d'expression visuelle et dramatique du cinéma. C'est ce que Grierson avait à l'esprit, de là sa définition du documentaire comme le "traitement créatif de l'actualité».

Défini de la sorte, le documentaire apparaît comme un hybride. Outil de connaissance et de compréhension d'une part, il remplit une importante fonction éducative. D'autre part, il éveille l'intelligence critique du spectateur, non pas directement, mais en faisant appel à son sens esthétique, à son imagination et à sa sensibilité; il a donc aussi un rôle à jouer dans le domaine de l'imaginaire.

Peu de documentaristes d'hier ou d'aujourd'hui s'aviseraient de contester la conception qu'avait Grierson de leur métier, et les 
exemples de l'art documentaire qui ont résisté au temps révèlent indubitablement un rapport harmonieux entre leur aspect informatif et leur forme artistique. Avec les années, cependant, toutes les formes de communication évoluent et, au Québec comme ailleurs, le documentaire a connu au cours du dernier demisiècle de nombreux avatars, donnant selon l'époque et les événements plus ou moins d'importance à l'un ou l'autre élément. On peut attribuer certains des changements qu'il a subis tantôt à une transformation générale de la sensibilité du public et du climat culturel, tantôt aux pressions plus directes du milieu politique et social ambiant. Trois stades importants marquent cette évolution : 1) la période griersonienne, jusqu'en 1960 environ ; 2) le cinéma direct, de 1960 à 1980 approximativement ; 3) ce que j'appelle le documentaire autoréflexif, qui commence vers la fin des années 70 . Si, dans ses plus brillantes manifestations, le documentaire griersonien a fait preuve d'une grande puissance artistique et nous a laissé quelques films qui ont marqué d'une pierre blanche l'histoire du cinéma, on note au cours de cette période une tendance du fonctionnel à l'emporter sur l'esthétique. Sur le plan de la forme, cette tendance se traduit par la transparence, typique du réalisme, qui cherche à effacer le médium pour ne laisser passer que le message. Sa caractéristique la plus évidente est le commentaire sur bande sonore, lu par un narrateur invisible, autorité incontestée, qui transmet au spectateur sa connaissance du sujet.

Dans ces conditions, rien de plus facile pour le documentaire griersonien que d'entrer dans le domaine télévisuel où régnait déjà un type de discours (celui du journalisme d'enquête), mettant au premier plan la présentation lucide des faits, l'omniscience et l'objectivité apparente, qui correspondait naturellement au sien.

Mais bien que le documentaire ait beaucoup gagné à fréquenter la télévision, devenue au fil des ans son principal débouché, il a dû se plier à différentes contraintes, dont un certain effet réducteur sur la forme et le langage n'a pas été la moindre des conséquences. Contrairement à ce que l'on pourrait penser de prime abord, les bulletins d'informations et les émissions d'affaires publiques adoptent une optique différente de 
celle du documentaire, précisément parce qu'ils n'aspirent pas à toucher l'imaginaire. Ils ont néanmoins avec lui un indéniable air de famille, ce qui a grandement facilité l'influence normalisatrice qu'a exercée le journalisme télévisé sur le documentaire didactique.

Au début des années 60, le documentaire griersonien s'était figé dans cette ressemblance, et on peut voir l'entrée en scène du cinéma direct comme une tentative pour redonner sa vitalité au genre, pour lui insuffler une nouvelle énergie créatrice.

Le cinéma direct procédait en partie d'une révolution technique : la caméra légère venait d'apparaître sur le marché, qui, en conférant aux réalisateurs une liberté d'action sans précédent, leur permettait de filmer spontanément et discrètement n'importe quelle scène. Plus important encore, son arrivée coïncidait avec l'explosion d'enthousiasme suscitée par la Révolution tranquille et avec le désir d'une nouvelle génération de réalisateurs de fixer sur pellicule les réactions du Québec, désormais libéré, devant ses nouvelles perspectives d'avenir. On ne saurait donc s'étonner de voir le documentaire s'éloigner radicalement du modèle griersonien. Rejetant ses visées didactiques et mettant au rancart la voix omnisciente qui impose son interprétation du sujet, le cinéma direct enregistre la réalité brute et s'efforce de laisser au spectateur l'illusion de reconstruire lui-même les événements à partir d'une profusion de détails.

La preuve la plus éclatante de la fécondité du cinéma direct, c'est que la plupart des documentaires québécois considérés comme des classiques du genre adoptent cette technique. Avec un peu de recul, cependant, on voit que le cinéma direct est tributaire d'une esthétique réaliste paradoxalement portée à se nier elle-même. Plus encore que le modèle griersonien, le cinéma direct est mû par l'idéal de la transparence. En effet, en raison justement de l'extrême souplesse de leur matériel, les réalisateurs jouent bien davantage que leurs prédécesseurs le rôle de simple témoin, dans lequel ils se cantonnent souvent. Si le documentaire péchait auparavant par excès de didactisme, le cinéma direct, lui, cédait à un certain mimétisme, se bornant à filmer la réalité en un flot continu, sans structure ni synthèse.

Au demeurant, l'emploi abondant que fait le cinéma direct de 
l'interview en champ montre à l'évidence son penchant pour l'observation pure. Malgré l'insistance que mettait Grierson sur l'importance de connaître ses semblables, le documentaire classique demandait rarement aux gens leur opinion. C'est au contraire le cinéma direct, grâce au matériel polyvalent dont disposaient les réalisateurs, qui s'est le plus rapproché de l'idéal griersonien. Tous les gens, sans égard à leur milieu social ni à leur situation, pouvaient parler librement devant la caméra, donc aux spectateurs et à leurs concitoyens. On peut donc y voir un véritable progrès pour la démocratie en ce qu'il permet aux gens ordinaires de s'exprimer publiquement. En même temps, il est clair que l'abus de l'interview fait très vite perdre toute forme au documentaire, car le réalisateur peut facilement laisser la bride sur le cou aux protagonistes et se borner à enregistrer passivement leurs témoignages. À cet égard comme à bien d'autres, le cinéma direct traduit l'influence de la télévision et tend lui aussi à se conformer à ses pratiques, renonçant ainsi à la distanciation créatrice qui devrait être la marque distinctive du documentaire.

Dans ce contexte, il n'est sans doute pas étonnant qu'au Québec, dans les années 80, le documentaire ait voulu s'affranchir du mimétisme pour expérimenter et innover en matière formelle. On peut voir dans ce mouvement, d'abord artistique, le désir de rétablir entre le fond et la forme l'équilibre sacrifié sur l'autel de la transparence. Après s'être refusés pendant 40 ans à tout esthétisme, les réalisateurs ont senti la nécessité de renverser la vapeur.

Cependant, cette nouvelle tendance s'appuie aussi sur des bases philosophiques plus profondes. Tous les courants de pensée contemporains remettent en question la vision de la réalité dont procèdent à la fois le documentaire griersonien et le cinéma direct. Les réalisateurs se sont rendu compte que la notion de réalité objective, indépendante de l'observateur, était indéfendable, et que même les observations effectuées dans la plus absolue neutralité ne pouvaient échapper à un processus de médiation, de manipulation et de construction. L'omniprésence du sujet supprime le fossé séparant le documentaire, axé en principe sur le réel et le vrai, du cinéma de fiction, censé reposer sur l'imagination et l'invention. Pour les documentaristes de la 
nouvelle vague, par conséquent, un spectateur ne peut comprendre le fait social qu'on lui présente que par l'intermédiaire de la conscience qui l'informe et l'interprète, au moyen de processus faisant appel autant à l'imagination qu'à l'observation.

Il s'ensuit que la plupart des nouveaux documentaires adoptent un ton fortement subjectif, voire un point de vue subjectif. La narration, bannie du cinéma direct, refait surface mais alors sous la forme d'une voix personnalisée ou d'un monologue intérieur. Le narrateur de La Fiction nucléaire, de Jean Chabot (1978), parle à la deuxième personne du singulier, mêlant à sa dénonciation de la politique du Canada en matière d'énergie nucléaire une sorte de dialogue avec lui-même qui rappelle le nouveau roman. Du même réalisateur, Le Futur intérieur (1982, en collaboration avec Yolaine Rouleau) et Voyage en Amérique avec un cheval emprunté (1987) sont construits autour de voyages en train ou en voiture, qui sont aussi de toute évidence des odyssées intérieures à la recherche de soi. Le Journal inachevé de Marilu Mallet (1982) est l'une des œuvres les plus remarquables sur l'adaptation des immigrants au Québec, précisément parce que le problème nous est présenté à travers la conscience de la réalisatrice et comme participant de toute son expérience subjective.

Ce recours à l'auto-interrogation méditative amène tout naturellement une structure complexe. Au lieu de la construction linéaire et séquentielle propre au modèle griersonien et au cinéma direct, les documentaires récents revêtent une forme éclatée comparable à celle de la littérature postmoderne. La discontinuité est accentuée par un mélange de "genres" dans de nombreux films, comme Le Grand Remue-ménage de Sylvie Groulx et Francine Allaire (1978). Bribes de chansons ou de danses, extraits de films, séquences d'animation et autres formes filmiques émaillent la matière documentaire. C'est ainsi qu'un même film se présente au spectateur comme une mosaïque de discours dont chaque parcelle exige de son imagination une réaction différente et dont l'interprétation le plonge dans un dilemme permanent.

Certaines œuvres sont parfois d'une telle complexité formelle que l'on peut à juste titre les qualifier d'autoréflexives. Elles font 
en sorte d'attirer l'attention sur leurs caractéristiques formelles propres et nous procurent d'autant plus de plaisir que nous saisissons mieux les procédés employés pour les réaliser. De tous les documentaires susceptibles de se classer dans cette catégorie, les plus parfaitement réussis sont peut-être L'Émotion dissonante (1984), de Fernand Bélanger, et Passiflora (1985), du même réalisateur, en collaboration avec Dagmar Gueissaz Teufel. Une comparaison de ces deux films avec Le Dernier Glacier (1984), de Jacques Leduc et Roger Frappier, permettra d'éclaircir certaines questions soulevées par le caractère expérimental caractéristique de cette nouvelle tendance.

Par son sujet - la fermeture de Schefferville, localité minière du Grand Nord québécois, et les conséquences désastreuses d'une pareille catastrophe économique sur le travail et la vie des habitants -, Le Dernier Glacier rejoint incontestablement les préoccupations traditionnelles du documentaire. Sa forme, cependant, l'en éloigne considérablement. Utilisant l'approche kaléidoscopique du nouveau documentaire, il entremêle des matériaux de natures différentes - séquences d'archives, extraits de documentaires et scènes de fiction - au point où le spectateur a parfois du mal à retrouver le fil de la narration. Il pousse plus loin encore l'expérimentation en juxtaposant souvent deux images différentes (qu'il fait aussi à l'occasion se mouvoir sur l'écran), obligeant ainsi le spectateur à lire deux messages simultanés entre lesquels il doit s'efforcer d'établir un lien.

Pourtant, si novateur soit-il, Le Dernier Glacier ne donne pas au spectateur l'impression d'être autoréflexif. Il dirige avec force l'attention sur les problèmes sociaux dont il traite, de sorte que le thème du film en transcende les aspects expérimentaux. C'est en partie que la substance documentaire, traitée dans le plus pur style du cinéma direct, est surtout constituée d'un mélange d'extraits de reportages et d'interviews en champ. De même, le récit fictif qui parcourt toute l'œuvre, où l'on voit un mariage détruit par suite de la fermeture de la mine, s'il est discontinu à l'intérieur de la structure générale, n'en est pas moins en soi parfaitement cohérent et conventionnel. L'intérêt du spectateur est d'autant mieux soutenu que les rôles du mari et de la femme sont joués par deux acteurs québécois bien connus. La participation au film de Michel Rivard, 
l'un des auteurs-compositeurs les plus populaires du Québec, qui tient un rôle mineur et interprète une chanson dramatique à un moment crucial, atteint le même résultat. Dans leur poignante simplicité, les paroles de la chanson assument en quelque sorte la même fonction que le commentaire omniscient du documentaire traditionnel en exprimant les sentiments de pitié et de colère que le film veut faire éprouver à l'auditoire face à la fermeture de la mine.

La présence de ces vedettes dans le film rappelle que l'une des raisons pour lesquelles le documentaire cherche à retrouver une certaine complexité de forme est qu'il revendique une place dans le royaume imaginaire qu'est le cinéma plutôt que dans le domaine informatif de la télévision. Ces dernières années, on est d'ailleurs parvenu à présenter en salle Le Dernier Glacier et plusieurs autres documentaires québécois de long métrage. Voilà pourquoi les innovations formelles de ce film ressortissent moins à l'autoréflexif qu'au spectaculaire. Il cherche essentiellement à allier les préoccupations traditionnelles du documentaire aux effets de séduction qu'exige le grand écran. À différents égards, il parvient à fondre le documentaire classique et le cinéma direct en un style qui correspond à la sensibilité de notre époque, notamment en procurant le plaisir esthétique d'un langage et d'une forme plus percutants. Son mérite est d'avoir su adapter la démarche du documentaire classique et du cinéma direct.

L'Émotion dissonante et Passiflora, documentaires de long métrage eux aussi, veulent également séduire le spectateur par l'impact de leur style visuel. L'ONF, producteur de L'Émotion dissonante, en a du reste extrait - dans le but évident d'intéresser le marché du vidéoclip - une courte séquence visuellement très travaillée où le groupe rock Offenbach interprète l'une de ses compositions. Certaines séquences de Passiflora, notamment un numéro de danse à claquettes vers la fin du film, mériteraient certainement le même sort. Ces deux films font preuve ainsi d'un sens aigu du spectacle. Il semble pourtant légitime d'affirmer qu'ils sont plus hermétiques et plus introvertis dans leur forme et leur climat que Le Dernier Glacier.

Le sujet qu'abordent les deux films est indéniablement d'intérêt public et relève des préoccupations traditionnelles du documentaire. L'Émotion dissonante porte sur la prise de drogue, 
en particulier de marijuana, chez les adolescents. Soutenant que la toxicomanie est moins dangereuse en soi que révélatrice d'un inquiétant manque de compréhension entre parents et enfants, le film définit son véritable propos comme étant la solitude et la détresse des jeunes. Même ainsi reformulé, le sujet s'inscrit nettement dans le contexte des problèmes sociaux qui ont toujours constitué le thème de prédilection du documentaire.

Le ton extrêmement satirique du début annonce dans Passiflora une démarche moins classique. Le film décrit les visites quasi simultanées du pape Jean-Paul II et du chanteur pop Michael Jackson à Montréal, qu'il traite essentiellement comme deux événements médiatiques exigeant les mêmes mesures massives de sécurité et suscitant la même ferveur populaire. C'est sur la visite du pape que porte surtout la satire, non seulement par sa juxtaposition ironique à celle de Jackson, mais aussi par la manipulation délibérée des images en vue de ridiculiser et de banaliser l'événement. Malgré ce ton persifleur, le film devient plus sérieux, et plus proche de L'Émotion dissonante, lorsqu'il commence à s'intéresser aux gens en détresse ou en marge de la société, aux jeunes surtout, exclus de tout ce cirque médiatique qui s'agite autour d'eux. Parmi eux, un couple d'amoureux qui cherche à obtenir un avortement, une femme âgée battue par son mari, mais aussi des homosexuels qui s'embrassent ostensiblement devant la caméra, et un travesti prostitué.

Après la satire du pape, la mise en lumière d'une sexualité différente passerait facilement pour un nouveau geste de provocation. Il n'empêche que le net changement d'orientation dans la seconde moitié du film dégage un message humain et généreux : ce sont les gens humbles et vulnérables, maltraités et proscrits, plutôt que les vedettes prestigieuses d'un cirque médiatique, qui méritent véritablement notre intérêt.

On peut même tirer du film deux interprétations opposées de la religion à partir des connotations ambivalentes associées à la passiflore, ou fleur de la Passion. Par ses propriétés sédatives, d'une part, elle suggère une religion au service du conformisme social - représentée par le pape. Symbole de la crucifixion, d'autre part, la passiflore évoque les malheureux, les victimes. Bien qu'à proprement parler aucun des marginaux ne porte de 
couronne d'épines, on peut voir en eux les victimes sacrificielles d'une société pharisaïque.

Moralement parlant, on peut donc dire que les deux films épousent l'objectif du documentaire traditionnel, qui est d'ébranler les certitudes dans lesquelles la société se complaît, de montrer des réalités cachées, d'élargir l'horizon social de notre compréhension et de notre compassion. Si ces bonnes intentions n'apparaissent pas d'emblée au spectateur, cela tient uniquement aux techniques employées. En effet, au lieu de procéder franchement et de manière didactique à l'analyse d'une situation sociale, les deux films cherchent à frapper l'imagination en faisant évoluer leurs personnages dans un univers le plus souvent fictif. La narration, l'interview et le reportage, moyens traditionnellement utilisés pour transmettre l'information, font en général place à des situations et à des événements mis en scène.

Il n'y a certes là rien de nouveau. Depuis ses débuts, le documentaire a recréé des situations réelles afin de rendre plus convaincante l'histoire qu'il raconte. Plus récemment, le genre du docu-drame a poussé beaucoup plus loin la dramatisation en donnant à des incidents réels la forme, l'intensité et les détails inventés d'une œuvre d'imagination. Dans le documentaire traditionnel, les éléments fictifs ont cependant toujours servi d'illustration. Selon le mot de Grierson, ce sont simplement des moyens de "donner vie" à des faits sociaux qui transcendent la fiction. Au contraire, dans L'Émotion dissonante et Passiflora, la fiction tend à délaisser cette fonction et à exister pour ellemême.

La caractéristique formelle la plus typique de ce processus est la nature fragmentaire des éléments fictifs. Alors que dans le docu-drame - auquel Le Dernier Glacier s'apparente sous ce rapport - l'histoire se suit de façon linéaire pour parvenir à son terme, nos deux films présentent des épisodes dramatiques incomplets et habituellement inachevés. Différents personnages les peuplent, dont nous ignorons presque tout et dont le sort nous reste inconnu, même après les avoir vus à plusieurs reprises. Leurs liens sont parfois surprenants et d'un arbitraire suspect : il se trouve ainsi que la femme battue de Passiflora est la mère du jeune hystérique dont de fugaces apparitions ponctuent le film. 
Des scènes représentées avec art - par exemple, celle du jardin botanique qui termine L'Émotion dissonante - sont sans relation évidente avec le reste de l'œuvre.

À cet égard, les éléments dramatiques des films nous placent dans une position analogue à celle du lecteur de nombreux romans postmodernes. Nous plongeons dans une atmosphère de fictionnalité diffuse sans que nous soit accordée la satisfaction de participer au déroulement logiquement ordonné de l'action. De la sorte, notre attention tend à se porter sur le mode d'expression plus que sur le sens exprimé. Notre plaisir à voir les films tient en bonne partie au fait que nous sommes conscients de la manière dont ils produisent sur nous leur effet.

Cela est vrai non seulement des éléments dramatiques, mais aussi des techniques cinématographiques, comme les mouvements de caméra et la profondeur de champ, qu'un emploi normal rendrait imperceptibles. Passiflora joue avec virtuosité du panoramique et du zoom pour établir des rapprochements entre les jeunes amants dans leur chambre, le cortège qui suit le pape et un autre couple dans la rue. Dans L'Émotion dissonante, une caméra hypnotique oscille entre des groupes attablés dans un café et l'action qui se déroule à l'extérieur, en même temps qu'elle élabore à partir des conversations un montage sonore de bribes de conversation que n'auraient renié ni Guillaume Apollinaire ni les dadaïstes du début du siècle. Car la bande sonore des deux films est tout aussi travaillée que l'agencement des images. Dans Passiflora, un autre "poème sonore" naît du chevauchement de conversations téléphoniques, tandis que les effets satiriques de la première partie proviennent surtout de ce que l'on a substitué des voix et de la musique discordantes au son original des documents d'archives montrant le "show» papal.

La pléthore de miroirs et de dispositifs réfléchissants dans chacun des films traduit l'insistance sur l'autoréflexion. Outre les véritables reflets dans des glaces, et sur les surfaces polies des couloirs du métro, de nombreux plans montrent des écrans de télévision, créant ainsi des images dans l'image. Bien qu'apparemment anodins, les extraits d'œuvres théâtrales jouées par des adolescents, dans L'Émotion dissonante, remplissent eux aussi la fonction autoréférente classique de la mise en abyme. 
Les séquences d'animation jouent un rôle particulièrement important à cet égard. Au service de la satire, d'abord, comme ce serpent espiègle qui traverse l'écran en ondulant pour aller défier le pape. Plus subtilement, elles visent à déstabiliser et à compliquer notre perception visuelle. Dans L'Émotion dissonante, les dessins sautillants de Pierre Hébert sont griffonnés en surimpression, produisant des images en perpétuel changement qui nous font voir la réalité sous forme de strates multiples. Les murs couverts de graffitis de Passiflora deviennent des panneaux coulissants qui, en se retirant, montrent à la façon d'un kaléidoscope les apparences derrière les apparences. Un effet analogue se produit lorsque ce que nous voyons à l'écran sous une forme réelle se trouve immédiatement transposé en animation. La juxtaposition de la réalité et de son reflet stylisé accentue le jeu des miroirs et fait ressortir la métamorphose des apparences et la relation formelle entre les images.

À tous ces points de vue, ces œuvres construisent non seulement un monde social, mais aussi un univers ludique relevant de l'imagination. Ce n'est pas pour rien qu'elles s'ouvrent toutes deux sur une note fantaisiste. Par la magie de l'animation, Passiflora fait jaillir des étincelles entre les doigts de jeunes adeptes de la break-dance, écho facétieux à la Création de Michel-Ange. Dans la même veine humoristique, on ne s'étonne pas que les panneaux de signalisation et une pieuse statue du Christ se mettent à bouger. Le premier personnage rencontré dans L'Émotion dissonante, une fille en habit de clown, réapparaît tout au long du film. Tantôt un singe à son bras, tantôt regardant à travers les fenêtres, se hissant au sommet des toits ou jouant de l'accordéon dans un centre commercial, elle incarne véritablement le "saltimbanque", le magicien-bateleur ambulant dont le regard transforme le quotidien en merveilleux. Les deux films sont ainsi parcourus par un fil ludique qui vient en alléger la gravité et se manifeste entre autres dans les scènes où un personnage danse, l'air concentré. À l'exubérant numéro de claquettes de Passiflora correspond une séquence de L'Émotion dissonante où, reléguée symboliquement à l'arrière-plan, une mère inquiète lit une lettre de sa fille qu'on suppose délinquante, tandis qu'au premier plan deux jeunes filles rythment le passage du 
temps en improvisant un ballet silencieux aux mouvements pleins de grâce.

De toute évidence, l'itinéraire décrit par les deux films nous conduit bien loin de l'étude pragmatique des questions sociales, domaine auquel nous associons le documentaire. Dans la scène du café de L'Émotion dissonante, il est révélateur que la caméra, interrompant ses lentes oscillations de table en table, s'immobilise un instant sur un tee-shirt orné de l'inscription "L'Émotion dissonante». On peut voir dans cette autoréférence littérale le retour sur soi qui caractérise généralement les deux films. Notre attention est attirée sur la richesse de leur organisation textuelle interne, et, indépendamment du sujet traité, nous sommes fascinés par la manière dont sont orchestrés leurs éléments sonores et visuels.

Les deux œuvres nous font aussi pénétrer beaucoup plus profondément dans l'imaginaire qu'on ne s'y attend de documentaires. Après en avoir terminé avec le pape, ce n'est pas par accident que Passiflora nous fait plonger dans les bas-fonds, au cœur de la nuit. La longue séquence nocturne, habile mélange de compassion sociale, de plaisirs dionysiaques et d'ambivalence sexuelle, fait au spectateur l'effet d'un voyage à travers le subconscient, se terminant par un retour à la surface, à la lumière du jour. De même, par les séquences se déroulant comme en rêve dans un bric-à-brac ou ce panoramique impitoyablement long sur un jeune garçon qui pense au suicide, l'imaginaire de L'Émotion dissonante explore la conscience troublée des adolescents.

On doit par conséquent se demander si ces films n'ont pas coupé toute attache avec le documentaire. Naturellement, la réponse dépend de la familiarité du spectateur avec les subtilités du cinéma. Bien que le cinéphile puisse aisément déceler leurs visées sociales sous leur complexité formelle, le spectateur moyen n'aura sans doute pas la patience de le faire. Même chez les critiques, il n'est pas rare d'entendre affirmer que les films ont poussé trop loin l'autoréflexion et sont devenus inabordables. Alors que Le Dernier Glacier, malgé la nouveauté de sa forme, parvient à préserver l'équilibre entre l'expression et le contenu qui caractérise le documentaire, ce rapport pourrait sembler 
avoir été trop radicalement bouleversé dans les deux autres œuvres.

S'il y a eu erreur, néanmoins, c'est certainement dans la bonne direction. Face à l'énorme développement des autres discours médiatiques, soumis au voisinage sans cesse plus étroit du journalisme télévisé, le documentaire ne peut survivre qu'en affirmant son caractère distinctif, lequel tient essentiellement à son aspect artistique et au plaisir esthétique qu'il procure. Le documentaire n'a effectivement pas le choix : il lui faut revenir au concept du traitement créatif de l'actualité et exploiter au maximum son potentiel formel.

Avec L'Émotion dissonante et Passiflora, comme avec d'autres films représentatifs de la nouvelle tendance autoréflexive, le documentaire a acquis une complexité de langage et une richesse de texture et de sens dont on ne trouve guère d'exemples dans toute son histoire. Voilà pourquoi ces deux films devraient demeurer, et devenir des points de référence par rapport auxquels on mesurera bon nombre d'œuvres à venir.

University of Stirling Traduit de l'anglais par Nicole Wanner en collaboration avec Anne Bienjonetti

\section{NOTE}

1 Cet article est d'abord paru sous le titre "The Self-conscious documentary in Quebec. L'Émotion dissonante and Passiflora" in Peter Easingwood, Konrad Gross and Wolfgang Kloos (ed), Probing Canadian Culture (Augsburg : AV-Verlag, 1991) pp. 225-234. Nous remercions l'éditeur et les responsables de l'ouvrage pour leur consentement à la publication de cet article dans sa traduction française.

2 Comme le producteur lui-même l'a proposé dans un communiqué de presse au moment du lancement du film. 